\title{
Study of optical characteristics of tin oxide thin film prepared by sol-gel method
}

\author{
SUMANTA KUMAR TRIPATHY ${ }^{1, *}$, BHABANI PRASAD HOTA ${ }^{2}$ and P V RAJESWARI ${ }^{1}$ \\ ${ }^{1}$ GVP College of Engineering (Autonomous), Madhurawada 530 048, India \\ ${ }^{2}$ Godavaris Mahavidyalaya, Banpur, Khurda 752 031, India
}

MS received 28 June 2012; revised 21 August 2012

\begin{abstract}
In this paper, we present details of preparation of tin oxide $\left(\mathrm{SnO}_{2}\right)$ thin film by sol-gel process. The film was synthesized on a glass (Corning 7059) plate by dip coating method. Here, we used tin (II) chloride as precursor and methanol as solvent. Optical characteristics and physical properties like refractive index, absorption coefficient and thickness of thin film were calculated from the study of transmission spectrum (wavelength vs transmission curve) data given by UV/VIS Spectrophotometer. Effect of number of coatings on transmittance and refractive index was also examined. It was observed that refractive index decreases with the number of coating and transmission value was more than $80 \%$ at wavelength greater than $450 \mathrm{~nm}$ in all cases. Structural analysis was studied by XRD measurement by using diffractometer which confirms tetragonal rutile structure of $\mathrm{SnO}_{2}$. Surface morphology was analysed from SEM micrograph and change in morphology on number of coat was discussed.
\end{abstract}

Keywords. Absorption coefficient; dip coating; spectrophotometer; transmittance.

\section{Introduction}

Now a days, the study and application of thin film technology has entirely entered into almost all the branches of science and technology due to brisk development of nanotechnology. The present paper deals with thin film of tin oxide which is more eye-catching to researchers due to its vast applications. Tin oxide thin films have some very beneficial properties, such as transparency for visible light, reflectivity for infrared light and a low electrical sheet resistance making them suitable for a wide variety of applications such as in transistors (Arnold et al 2003), photovoltaic cell (Cachet et al 1997), gas sensors (Butta et al 1992), protective and wear-resistant coating on glass containers (Nakagawa et al 1997), Infrared reflectors for glass windows (Lindner 1988), etc. The gas sensing properties of tin oxide thin films have been performed for different gases like $\mathrm{CO}, \mathrm{NO} x, \mathrm{H}_{2} \mathrm{~S}, \mathrm{H}_{2}$, $\mathrm{CH}_{4}$ and $\mathrm{CNG}$ etc. (Varghase and Malhotra 1998; Baik et al 2000; Niranjan and Mulla 2003). Many methods are used to synthesize doped or undoped tin oxide films such as thermal evaporation (Comini et al 2002; Vaishnav et al 2005), chemical vapour deposition (Gorley et al 2005; Mamazza et al 2005), magnetron co-sputtering (Yoo et al 2005; Jeorg et al 2006), laser pulse evaporation (Yang and Cheung 1982; Hui et al 2002), spray pyrolysis (Lane et al 1992; Pirmoradi et al 2011) and sol-gel (Culha et al 2009). Out of all the above methods, sol-gel technique plays an important role due to several advantages such as easy control on film thickness with a high porosity area which can improve efficiency

\footnotetext{
*Author for correspondence (sktripathy2009@gmail.com)
}

of the sensors, low processing cost, greater homogeneity and more purity. Using this method, we can prepare optical quality films with desired refractive index and small thickness.

Tin oxide is a tetragonal $n$-type semiconductor having high bandgap energy $(\approx 3.6 \mathrm{eV})$ (Kilic and Zunger 2002). It is more transparent in the region of visible spectrum due to high bandgap and having high electrical conductivity due to free electrons in oxygen vacancy holes. Due to the above reasons and large surface area on thin films, tin oxide and doped tin oxide thin films are of greater significance for the researchers.

Generally, there are three methods used in sol-gel technique. They are spin coating, dip coating and spray coating. In this study, dip coating method was applied. Starting from tin (II) chloride which was preferred due to low cost as precursor, methanol as solvent and glacial acetic acid as chelating agent, a transparent solution was prepared and $\mathrm{SnO}_{2}$ thin film was synthesized on a glass substrate (Corning 7059) by novel sol-gel dip coating technique. This was the better choice of sol-gel method than the alkoxide or tin (IV) chloride due to the cost factor and availability. The main purpose of this presentation was to study optical properties of tin oxide thin film from the interference fringes of the transmission spectrum which is carried out by the equipment ELICO UV/VIS spectrophotometer (Model, SL-159) in the wavelength range 300-1000 nm. Effect of number of coatings on thickness, refractive index and transmittance was also analysed.

Structural analysis of the films was carried out by XRD measurement using SIEMENS diffractometer (Model D5000). The study confirms tetragonal rutile structure of $\mathrm{SnO}_{2}$. Surface morphology was examined from SEM 
micrographs by using scanning electron microscope (Model, Philips XL 30).

\section{Experimental}

\subsection{Preparation of solution}

$1 \mathrm{~g}$ of anhydrous tin (II) chloride $\left(\mathrm{SnCl}_{2}\right)$ was dissolved in $50 \mathrm{ml}$ of methanol $\left(\mathrm{CH}_{3} \mathrm{OH}\right)$ with $1 \mathrm{~g}$ glacial acetic acid $\left(\mathrm{CH}_{3} \mathrm{COOH}\right)$ in a conical flask. The solution was continuously stirred by a magnetic stirrer for $45 \mathrm{~min}$ at NTP to get a clear homogeneous solution.

\subsection{Cleaning substrate}

Before coating on the glass substrate (Corning 7059), the substrates were thoroughly cleaned with cleaning liquid soap and then with acetone to remove organic particles on the surface and then washed with distilled water. To prevent local hydrolysis, the substrates were then soaked with TEA diluted isopropyl alcohol for $10 \mathrm{~min}$ and then dried.

\subsection{Dipping details}

Now one substrate was dipped in the prepared solution by hand at a speed of $\sim 20 \mathrm{~cm} / \mathrm{min}$ and moved out with the same speed. Here, we have maintained the same speed of dipping and withdrawal to get uniform thickness. Generally, physical properties of films prepared by sol-gel method depends on solution concentration, heat treatment temperature and withdrawal speed. According to Schroeder (1969), $d \propto v^{2 / 3}$, where ' $d$ ' is thickness of the film and ' $v$ ' the withdrawal speed. Here, the authors would like to mention that since, it is operated by hand the speed might not be uniform but precautions were taken to make the speed nearly uniform. However, this method can be improvized by using gears and electric motor to make the speed constant.

\subsection{Heat treatment}

The coated glass substrate was dried at $150{ }^{\circ} \mathrm{C}$ in a muffler furnace for $1 \mathrm{~h}$ to remove other products. Then the substrate was heat treated at $300{ }^{\circ} \mathrm{C}$ for about $15 \mathrm{~min}$. The above procedure was repeated for a number of times to get the desired thickness (1st substrate, one time, 2nd substrate two times, 3rd substrate, three times,..., 8th substrate, eight times). In this dipping process, there was two-sided coat on the substrate. For optical transmission measurements the film on one side of the substrate was required, so other side coat was removed by means of a sharp edge. While removing the coat precautions were taken, so that the other side coat would not be disturbed at all.

Then finally heat treatment was carried out on each substrate at $500{ }^{\circ} \mathrm{C}$ for $90 \mathrm{~min}$ in a muffler furnace in air.

Figure 1 shows schematic diagram of preparation of tin oxide thin film by sol-gel process.

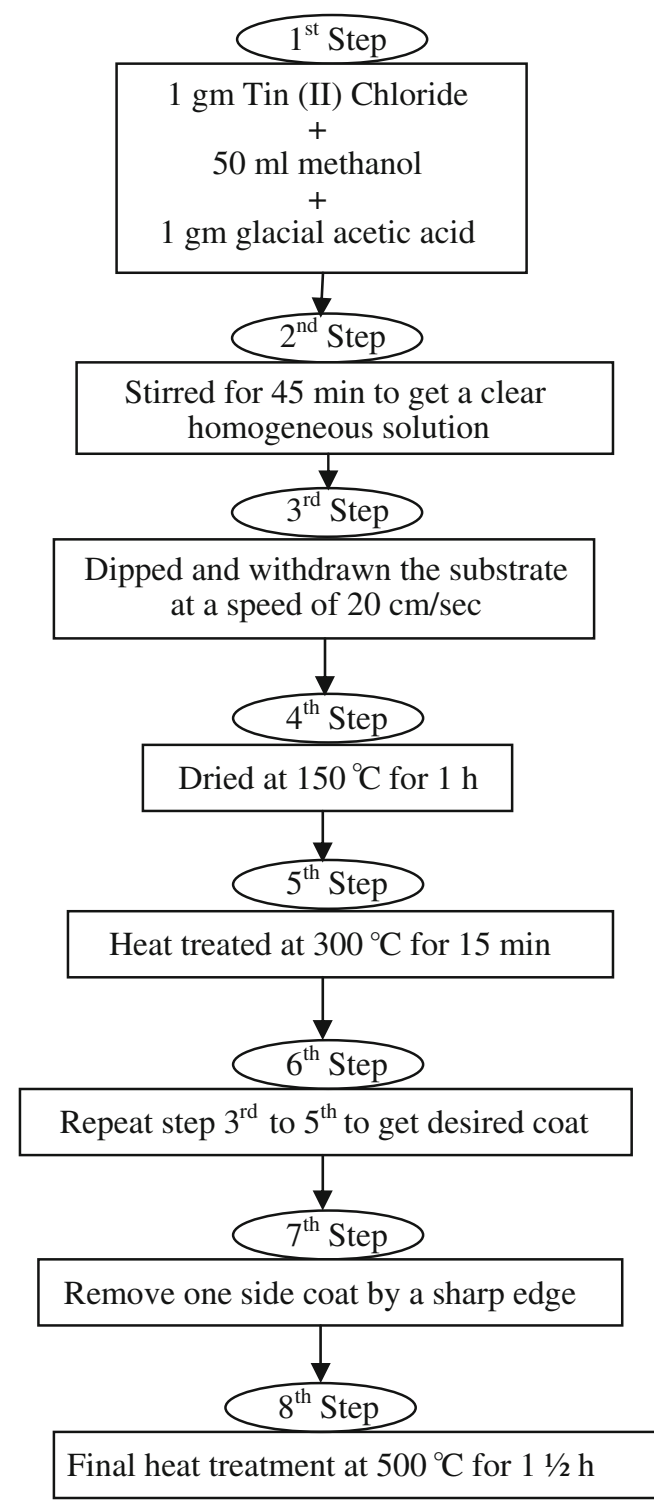

Figure 1. Schematic diagram.

\subsection{Optical characterization}

Optical characterization was studied from transmission $\%$ vs wavelength curve which was plotted from the data obtained from transmission spectrum analysis of the film by ELICO UV/VIS spectrophotometer, Model, SL 159 in the wavelength range $300-1000 \mathrm{~nm}$. From the figure 2, it is clear that the surface quality and homogeneity of thin film was excellent.

\subsection{Theory of thickness measurement}

In this study, the refractive index and thickness of the film were calculated using the envelop method (Manifacier et al 1976). From the theory of interference in thin films the fundamental equation for the interference is:

$$
2 n d \cos r=m \lambda,
$$




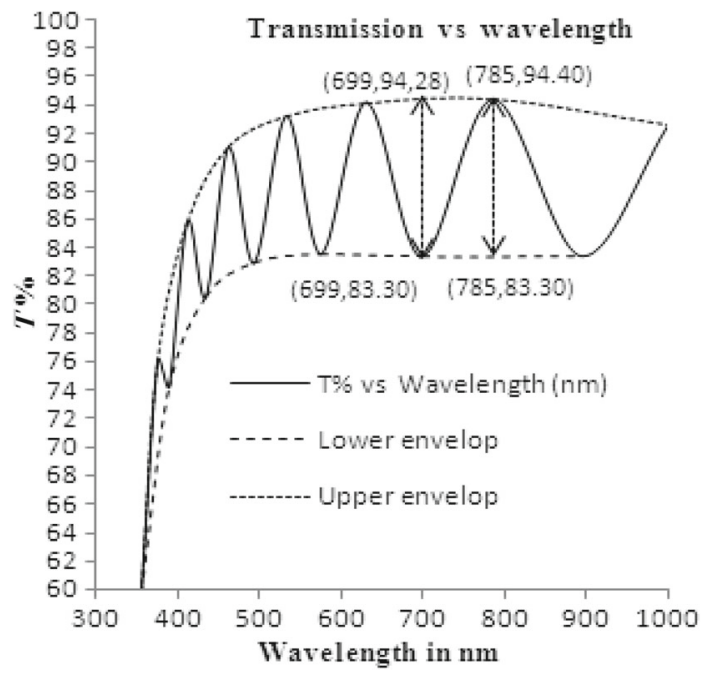

Figure 2. Transmission vs wavelength (nm) curve in wavelength range $300-1000 \mathrm{~nm}$ of 8 th coated film.

where $m$ is an integer for maxima and half an integer for minima, $n$ the refractive index of the film, $\lambda$ the wavelength and $d$ the thickness of the film.

For normal incidence, $r=0^{\circ}$, the equation for the interference pattern becomes:

$$
2 n d=m \lambda \text {. }
$$

Now, the transmission coefficient (Swanepoel 1983) is:

$$
T=\frac{A_{\mathrm{x}}}{B-C_{\mathrm{x}} \cos \theta+D_{\mathrm{x}}^{2}},
$$

where $A, B, C$ and $D$ are the constants given by:

$$
\begin{aligned}
& A=16 n^{2} \mu, \quad B=(n+1)^{3}\left(n+\mu^{2}\right), \\
& C=2\left(n^{2}-1\right)\left(n^{2}-\mu^{2}\right), \\
& D=(n-1)^{3}\left(n-\mu^{2}\right), \\
& \varphi=\text { phase difference }=\frac{4 \pi n d}{\lambda}, \\
& x=\text { absorptance }=e^{-\alpha d}, \\
& \alpha=\text { absorption coefficient }=\frac{4 \pi k}{\lambda}, \\
& k=\text { propagation wavevector for wavelength } \lambda .
\end{aligned}
$$

If $T_{\mathrm{u}}$ and $T_{1}$ be the transmission maximum at upper envelop and transmission minimum at lower envelop for a particular wavelength $\lambda$ then:

$$
T_{\mathrm{u}}=\frac{A_{\mathrm{x}}}{B-C_{\mathrm{x}}+D_{\mathrm{x}}^{2}}
$$

and

$$
T_{1}=\frac{A_{\mathrm{x}}}{B+C_{\mathrm{x}}+D_{\mathrm{x}}^{2}} .
$$

Since thin film of $\mathrm{SnO}_{2}$ is not a completely transparent region, therefore, $\alpha \neq 0$ and $x<1$ (Swanepoel 1983).

Now,

$$
\frac{1}{T_{1}}-\frac{1}{T_{\mathrm{u}}}=\frac{B+C_{\mathrm{x}}+D_{\mathrm{x}}^{2}-B+C_{\mathrm{x}}-D_{\mathrm{x}}^{2}}{A_{\mathrm{x}}}=\frac{2 C}{A} .
$$

Putting the values of $C$ and $A$ from (4a) in (7):

$$
\frac{1}{T_{1}}-\frac{1}{T_{\mathrm{u}}}=\frac{T_{\mathrm{u}}-T_{1}}{T_{\mathrm{u}} T_{1}}=\frac{\left(n^{2}-1\right)\left(n^{2}-\mu^{2}\right)}{4 n^{2} \mu} .
$$

Solving (8) we have:

$$
n=\left\{N+\left(N^{2}-\mu^{2}\right)^{1 / 2}\right\}^{1 / 2},
$$

where

$$
N=2 \mu \frac{T_{\mathrm{u}}-T_{1}}{T_{\mathrm{u}} T_{1}}+\frac{\mu^{2}+1}{2},
$$

where $n$ is the refractive index of thin film $\mu$ the refractive index of the substrate, $T_{\mathrm{u}}$ and $T_{1}$ the transmission maximum at upper envelop and transmission minimum at lower envelop for a particular wavelength $\lambda$.

If $n_{1}$ and $n_{2}$ be the refractive index of thin film at maxima (for wavelength $\lambda_{1}$ ) and corresponding minima (for wavelength $\lambda_{2}$ ) where phase difference is $\pi$ and $\varphi_{1}, \varphi_{2}$ be the phase angle at maxima and minima then from equation,

$$
\varphi=\frac{4 \pi n d}{\lambda} .
$$

We have

$$
\varphi_{1}=\frac{4 \pi n_{1} d}{\lambda_{1}}
$$

and

$$
\varphi_{2}=\frac{4 \pi n_{2} d}{\lambda_{2}}
$$

and also $\varphi_{1}-\varphi_{2}=\pi$.

So,

$$
\begin{aligned}
\pi & =\frac{4 \pi n_{1} d}{\lambda_{1}}-\frac{4 \pi n_{2} d}{\lambda_{2}}=4 \pi d \frac{\left(n_{1} \lambda_{2}-n_{2} \lambda_{1}\right)}{\lambda_{1} \lambda_{2}} \\
& \Rightarrow d=\left|\frac{\lambda_{1} \lambda_{2}}{4\left(n_{1} \lambda_{2}-n_{2} \lambda_{1}\right)}\right| .
\end{aligned}
$$

However, if we consider consecutive two maxima whose phase difference is $2 \pi$, then

$$
d=\left|\frac{\lambda_{1} \lambda_{2}}{2\left(n_{1} \lambda_{2}-n_{2} \lambda_{1}\right)}\right| .
$$

In this paper, we have used (11) to find the width of thin film due to the fact that $\lambda_{1}$ and $\lambda_{2}$ are taken from the minima and subsequent maxima. 


\section{Results and discussion}

\subsection{Measurement of thickness}

Here, we have mathematically calculated the thickness of the 8th coated film.

From figure 2 for maxima:

$$
\lambda_{1}=785 \mathrm{~nm}, T_{\mathrm{u}}=0.944, T_{1}=0.833 \text { and } \mu=1.53,
$$

Then

$$
N_{1}=2 \mu \times \frac{T_{\mathrm{u}}-T_{1}}{T_{\mathrm{u}} \times T_{1}}+\frac{\mu^{2}+1}{2}=2 \cdot 10,
$$

and

$$
n_{1}=\left\{N_{1}+\left(N_{1}^{2}-\mu^{2}\right)^{1 / 2}\right\}^{1 / 2}=1.881 .
$$

From figure 1, for minima:

$$
\lambda_{2}=699 \mathrm{~nm}, T_{\mathrm{u}}=0.9428, T_{1}=0.833 \text { and also } \mu=1.53,
$$

Then

$$
N_{2}=2 \mu \times \frac{T_{\mathrm{u}}-T_{1}}{T_{\mathrm{u}} \times T_{1}}+\frac{\mu^{2}+1}{2}=2.097,
$$

and

$$
n_{2}=\left\{N_{2}+\left(N_{2}^{2}-\mu^{2}\right)^{1 / 2}\right\}^{1 / 2}=1.879 .
$$

Using the relation:

$$
d=\left|\frac{\lambda_{1} \lambda_{2}}{4\left(n_{1} \lambda_{2}-n_{2} \lambda_{1}\right)}\right| .
$$

We have $d=856 \cdot 32 \mathrm{~nm}$.

In this experiment, we got the average refractive index of thin film as 1.88 , which is nearly the same with the result obtained by Manifacier et al (1977).

\subsection{Film thickness vs number of coatings}

After each coating, the sample was studied for its optical characterization and from the transmission vs wavelength graph average refractive index and thickness were measured by the above mentioned formula. The result of measurements of thickness and refractive index for even coat are shown in table 1.

Table 1. Number of coatings vs thickness and $n$.

\begin{tabular}{lcc}
\hline $\begin{array}{l}\text { No. of } \\
\text { coatings }\end{array}$ & $\begin{array}{c}\text { Film thickness } \\
(\mathrm{nm})\end{array}$ & $\begin{array}{c}\text { Average refractive } \\
\text { index }(n)\end{array}$ \\
\hline 2 & 212.13 & 2.56 \\
4 & 429.65 & 2.40 \\
6 & 645.98 & 2.07 \\
8 & 856.32 & 1.88 \\
\hline
\end{tabular}

Looking at the data presented in table 1, one can see that how thickness of thin film varies with number of coatings. It follows that refractive index of $\mathrm{SnO}_{2}$ film decreases monotonically with increase of thickness due to less porosity in the film. Thus, it may be believed that the grain size of the film of less thickness is more. The higher value of refractive index may be due to the increase of inhomogenity and surface roughness of the films of less number of coat applications.

Figure 3 shows graph between number of coatings and thickness. The curve is nearly linear. It indicates that for each coat, thickness increases by 105-109 nm. The thickness of each coat depends on the dilution of the precursor solution as well as the firing temperature.

\subsection{Transmission\% vs number of coatings}

Figure 4 shows variation of transmittance with the number of coatings. Here we have plotted the curve of transmittance vs wavelength for even number of coatings (2nd, 4th, 6th and 8th).

It was found that as the thickness increases the transmission\% decreases. It may be due to less porosity and small grain size in thick films. It is also clear that transmission

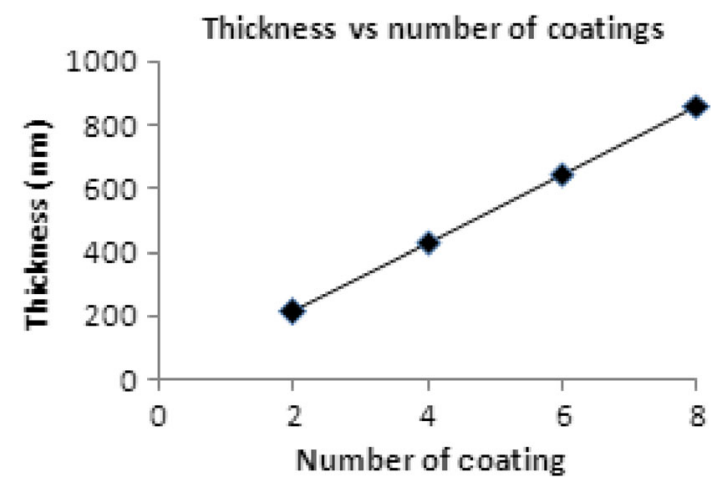

Figure 3. Thickness vs number of coatings.

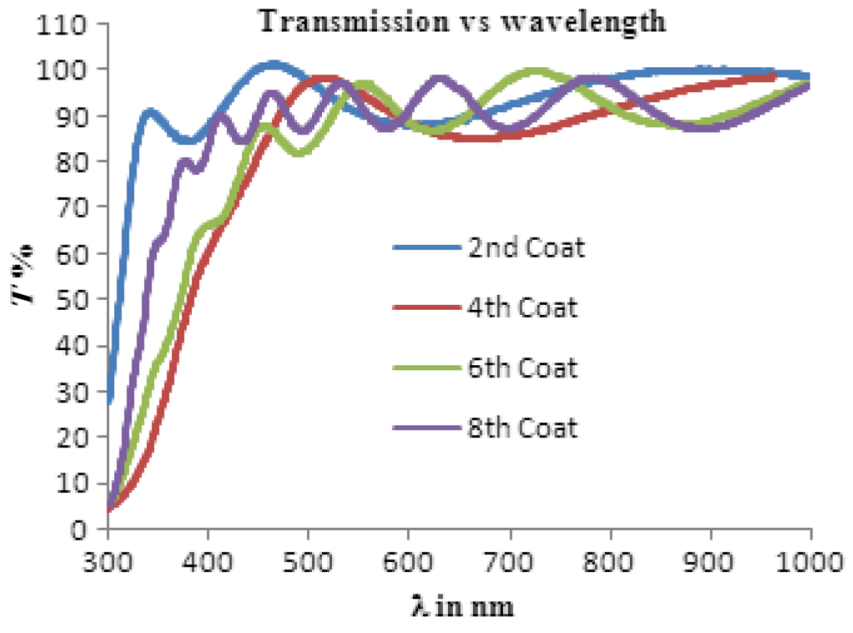

Figure 4. $T \%$ vs number of coatings. 
values were more than 0.80 at wavelength $>450 \mathrm{~nm}$ in all the cases. The spectra of second and fourth coat films contain less number of peaks which may be due to inhomogenety and surface roughness of the films.

From the transmission spectra, the absorption coefficient was calculated by using the formula (Tarey and Raju 1985):

$$
\dot{a}=\frac{1}{d} \ln \left(\frac{1}{T}\right),
$$

where $d$ is the thickness of the film and $T$ the optical transmission. The calculated absorption coefficient was about $10^{4} \mathrm{~cm}^{-1}$ for 8 th coat film which may be suitable for a transparent conducting film. However, ultra thin tin oxide film may not act as transparent conducting film due to the fact that resistance of film increases with decrease in film thickness.

\subsection{Structural analysis}

Figure 5 shows XRD pattern of $\mathrm{SnO}_{2}$ thin film for different coats. XRD measurement was carried out by Siemens Diffractometer Model-D 5000 using $\mathrm{CuK} \alpha$ having wavelength $\lambda=0.1540 \mathrm{~nm}$ radiation with a diffraction angle $10-70^{\circ}$.
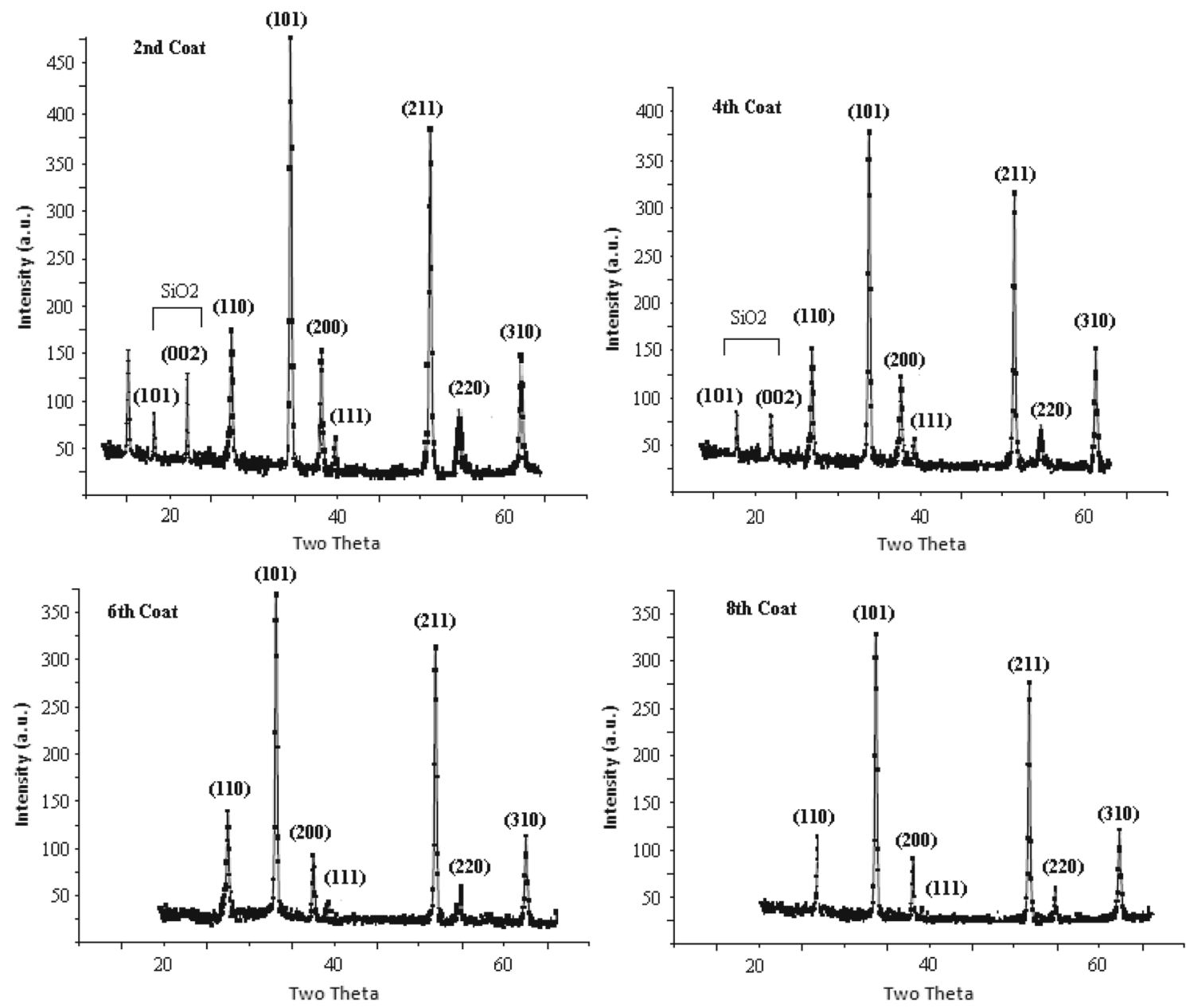

From figure 5, it was observed that in all cases welldefined sharp diffraction peaks are obtained nearly at same angle of $2 \theta$ which may be considered to be the crystalline tetragonal rutile structure of $\mathrm{SnO}_{2}$ (JCPDS Card No. 880287). The well-defined peaks which match the standard interplanar spacing JCPDS card no. $88-0287$ is given as $26 \cdot 6^{\circ}$ for (110) plane, $33.9^{\circ}$ for (101) plane, $38^{\circ}$ for (200) plane, $39^{\circ}$ for (111) plane, $51.8^{\circ}$ for (211) plane, $54.8^{\circ}$ for $(220)$ and $61.9^{\circ}$ for (310) plane. Since XRD peaks are very narrow and sharp, it indicates higher crystalline quality of $\mathrm{SnO}_{2}$ film. The (101) peak has the largest intensity in all cases, but others like (101), (110), (200), (220) and (310) are clearly identified. Since the intensity of (101) plane is more, it may be believed that the preferential growth along (101) direction of $\mathrm{Sn}$ forms an interstial bond with oxygen and exists as rutile $\mathrm{SnO}_{2}$. Again, we can observe that the intensity of peaks decreases with increase in the number of coatings which may be due to the large grain size and more porosity for less thick film. Ultimately, refractive index of film is increased, when thickness decreases as shown in table 1 . Since, we did not find any reflection peaks from the impurities in XRD spectra, high purity of the product may be confirmed.

Phase identification of $\mathrm{SnO}_{2}$ film on the glass substrate was clearly observed from XRD spectra. $\mathrm{SiO}_{2}$ crystalline

Figure 5. XRD patterns of $\mathrm{SnO}_{2}$ thin film. 

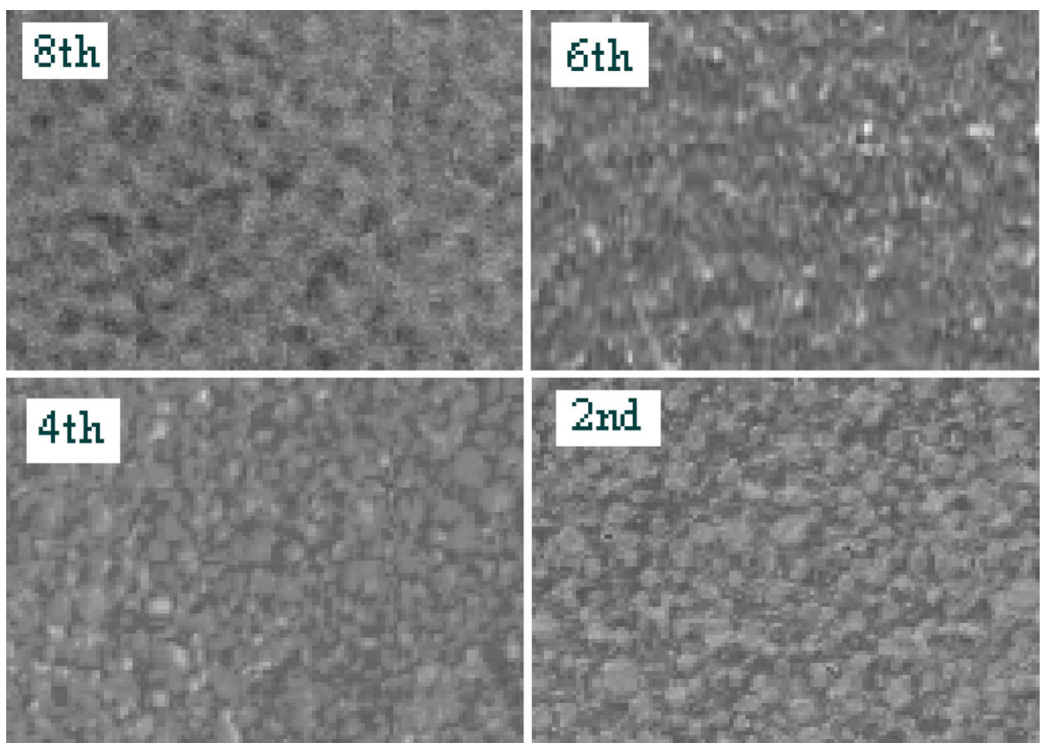

Figure 6. SEM images of $\mathrm{SnO}_{2}$ film for different number of coat applications.

phase peaks were observed in the film with 2 nd and 4 th coats whereas these peaks were absent in the film with 6th and 8th coats. The two extra peaks correspond to $16.5^{\circ}$ for (101) and $22 \cdot 3^{\circ}$ for (002) planes, respectively of $\mathrm{SiO}_{2}$. The extreme left peak in the film of 2 nd coat at $10.4^{\circ}$ was unidentified which may probably be due to some impurities. Thus, structural configuration was changed with a number of coatings due to which $\mathrm{SiO}_{2}$ crystalline phases were seen in the spectra of films with less number of coat application.

XRD spectra was analysed with Gaussian function where FWHM was determined. By using Debye-Scherrer formula (Jeorg et al 2006):

$$
D=\frac{0 \cdot 9 \lambda}{\beta \cos \theta}
$$

where $D$ is the mean grain size, $\beta$ the FWHM of the observed peak, $\lambda$ the wavelength of $\mathrm{X}$-ray used for diffraction and $\theta$ the angle of diffraction. Using the above formula, average grain size of the deposited film was calculated as 50.09, $49.31,48.54$ and $47.34 \mathrm{~nm}$ for $2 \mathrm{nd}$, 4th, 6th and 8th coating films, respectively. This clearly speaks that the grain size increases with decrease in thickness. Hence, roughness increases and ultimately refractive index increases, which confirms the result from optical properties as discussed in $\S 3.2$.

\subsection{Morphological analysis}

SEM images of $\mathrm{SnO}_{2}$ thin film deposited on glass substrate was shown in figure 6 for different number of coat applications. SEM measurement was carried out by scanning electron microscope Model-Philips XL 30. In this figure, effect of number of coatings on morphology of $\mathrm{SnO}_{2}$ film was observed. Surface morphology of 8th coating depicts coating islands are formed due to increase in thickness. However, cracks are not predominantly present as observed by Culha et al (2009) for the film thickness of $1015 \mathrm{~nm}$. The formation of coating islands may be due to the removal and combustion of organic group during frequent heat treatment and also may be due to the release of tensile force on film coat.

SEM micrographs show agglomeration of the grain particles in 2nd, 4th and 6th coat films. The surface roughness increases as the thickness decreases. From SEM images, it was clear that microstructural properties as well as thickness of the film changes with number of coat. SEM micrographs of 6th, 4th and 2nd coats contain dome-like structures and size of the domes increases as number of coat decreases, i.e. as the thickness increases. This dome-like structures may be believed as the top surfaces of the grains of the film. Since the size of domes increases with decrease in thickness, so, it may be concluded that the grain size of the film having less thickness is bigger than the grain size of the film of more thickness which was already discussed in \$3.2.

Analysing the data of XRD and SEM measurements for grain size, we observed there was a marked difference in grain size calculated by XRD and SEM methods especially for 8 th and 6th coating films. The grain size calculated by XRD method was smaller than that estimated by using SEM images. It was observed that for 8th coating $\mathrm{SnO}_{2}$ film, the grain size varies from 47 to $52 \mathrm{~nm}$ whereas the average grain size calculated by XRD was $47.34 \mathrm{~nm}$. As far as $\mathrm{SnO}_{2}$ film, of $2 \mathrm{nd}$ and 4 th coats are concerned, the difference in grain size is $0.2 \mathrm{~nm}$. This difference in grain size may be due to the presence of mechanical strains which are unevenly distributed over the film thickness. This also may be due to $\mathrm{SnO}_{2}$ crystallites' inclination to twinning (Pan and Zheng 1997). It was found that many crystallites of $\mathrm{SnO}_{2}$ are multiple twinned with twinning planes, parallel to one of (101) lattice plane. 


\section{Conclusions}

Tin Oxide films were synthesized on glass substrate by solgel (dip coating) method. Optical characteristics of thin film were determined from the transmittance spectra in UV-VIS region using the envelop method. It was observed that thickness of the film increases nearly $105-109 \mathrm{~nm}$ for each coat. The transmittance of the film was measured with a number of applications. It was observed that transmittance decreases as the number of coatings increases and transmission value were more than 0.80 at wavelength $>450 \mathrm{~nm}$ in all cases. If the dilution of the precursor solution is more, thickness of each coat is less and obviously transmittance will be more. From XRD study, it was concluded that, the structural configuration changes with number of coatings due to which $\mathrm{SiO}_{2}$ crystalline phases were seen in the spectra of films with less number of coat applications. SEM images show surface roughness of the film. As sensing of gas by thin film will be more in case of more surface roughness, the obtained experimental results can be suitably used for gas sensors by taking $\mathrm{SnO}_{2}$ thin films of thickness $<450 \mathrm{~nm}$. Both XRD and SEM results confirm that the product is a tetragonal rutile structure.

\section{Acknowledgements}

Authors are grateful to the management of GVP College of Engineering (Autonomous), Visakhapatanam for providing Laboratory facilities. Authors are also thankful to Prof. T S N Somayaji, Director of Research (Science) for his support to carry out the research work.

\section{References}

Arnold M S, Avouris P, Pan Z W and Wang Z L 2003 J. Phys. Chem. B107 659

Baik N S, Sakai G, Miura N and Tamajoe N 2000 Sensor Actuator. B63 74
Butta N, Cinquegrani L, Mugno E, Tagliente A and Pizzini S 1992 Sensor Actuator. B6 253

Cachet H, Bruneaux J, Folcher G, Levy-Clement C, Vard C and Neumann-Spallart M 1997 Solar Energ. Mater Solar C. 46101

Comini E, Faglia G, Sberveglieri G, Pon Z and Wang Z L 2002 Appl. Phys. Lett. 811869

Culha O, Ebeoglugil M F, Birlik I, Celik E and Toparli M 2009 J. Sol-Gel Sci. Technol. 5132

Gorley P M, Khomyak V V, Bilichuk S V, Orletsky I G, Hovly P P and Grechko V O 2005 Mater. Sci. Eng. B118 160

Hui F, Miller T M, Magruder R M and Weller R A 2002 J. Appl. Phys. 916194

Jeorg J, Choi S P, Hong K J, Song H J and Park J S 2006 J. Korean Phys. Soc. 48960

Kilic C and Zunger A 2002 Phys. Rev. Lett. 88 095501(1)

Lane D W, Coath J A, Rogers K D, Hunnikin B J and Beldon H S 1992 Thin Solid Films 221262

Lindner G H 1988 US Patent 4737388

Mamazza R, Morel D L and Ferekider C S 2005 Thin Solid Films 48426

Manifacier J C, Gasiot J and Fillard J P 1976 J. Phys. E: Sci. Instrum. 91002

Manifacier J C, Demurcia M and Fillard J P 1977 Thin Solid Films 41127

Nakagawa M, Amano T and Yokokura S 1997 J. Non-Cryst. Solids 218100

Niranjan R S and Mulla I S 2003 Mater. Sci. Eng. B103 103

Pan X and Zheng J G 1997 Mat. Res. Soc. Symp. Proc. 47287

Pirmoradi H, Malakootikhah J, Karimipour M, Ahmadpour A, Shahtahmasebi N and Koshky F E 2011 Middle-East J. Sci. Res. 8253

Schroeder H 1969 Physics of thin films (New York: Academic Press) Vol. 5, p. 87

Swanepoel R 1983 J. Phys. E: Sci. Instrum. 161214

Tarey R D and Raju T A 1985 Thin Solid Films 128181

Vaishnav V S, Patel P D and Patel N G 2005 Thin Solid Films 49094

Varghase O K and Malhotra L K 1998 Sensor Actuator. B53 19

Yang H T and Cheung Y T 1982 J. Cryst. Growth 56429

Yoo K S, Park S H and Karg J H 2005 Sensor Actuator. B180 159 